\title{
Effect of Celecoxib on Surgical Site Inflammation after Total Knee Arthroplasty: A Randomized Controlled Study
}

\author{
Xun Xu Weilin Sang Yu Liu Libo Zhu Haiming Lu Jinzhong Ma \\ Department of Orthopedics, Shanghai General Hospital, Shanghai, China
}

\section{Significance of the Study}

- Celecoxib has a significant inhibitory effect on postoperative aseptic inflammatory reaction, especially in the early period after total knee arthroplasty. It can also promote early rehabilitation of knee joint function of patients.

\section{Keywords}

Total knee arthroplasty - Aseptic inflammatory reaction •

Selective COX-2 inhibitor · Inflammation index

\begin{abstract}
Objective: To evaluate the anti-inflammatory effectiveness of celecoxib and its effect on the rehabilitation of joint function after total knee arthroplasty. Methods: 72 patients presented between 2016 and 2017 and were divided into two groups. The experimental group was given $200 \mathrm{mg}$ celecoxib twice daily with tramadol hydrochloride $50 \mathrm{mg}$ twice daily (as required); the control group was given tramadol hydrochloride $50 \mathrm{mg}$ twice daily for 6 weeks from the first day after total knee arthroplasty. Skin temperature around the knee was measured 1 day before surgery, on postoperative days 1 and 3, and at weeks 1, 2, and 6. Inflammatory markers (white blood cell count, C-reactive protein, erythrocyte sedimentation rate, and interleukin-6) were measured preoperatively, on postoperative day 3, and at weeks 1 and 6. Knee
\end{abstract}

\begin{tabular}{ll}
\hline KARGER & (c) 2018 The Author(s) \\
Published by S. Karger AG, Basel & Karger \\
E-Mail karger@karger.com & $\begin{array}{l}\text { This is an Open Access article licensed under the Creative Commons } \\
\text { Attribution-NonCommercial-4.0 International License (CC BY-NC) } \\
\text { (http://www.karger.com/Services/OpenAccessLicense), applicable to } \\
\text { the online version of the article only. Usage and distribution for } \\
\text { commercial purposes requires written permission. }\end{array}$
\end{tabular}

Society Score was recorded preoperatively and at postoperative weeks 1, 2, and 6. Results: Except for preoperative skin temperature, the recorded skin temperatures of the experimental group were significantly different compared to those of the control group ( $p=0.001,0.024,0.030,0.041$, 0.047 , respectively). Levels of C-reactive protein were significantly different at the $1 \mathrm{st}$ and the 6th week after surgery, differing by $19.3 \pm 4.64 \mathrm{mg} / \mathrm{L}(p<0.001)$ and $2.6 \pm 0.92 \mathrm{mg} / \mathrm{L}$ $(p=0.006)$. Levels of interleukin-6 showed a significant difference of $6.61 \pm 2.36 \mathrm{pg} / \mathrm{mL}(p=0.007)$ at the 1 st week after surgery. Until the 6th week after surgery, the erythrocyte sedimentation rate in the experimental group and the control group differed by $17 \pm 4.6 \mathrm{~mm} / \mathrm{h}(p=0.001)$. Conclusions: Celecoxib has a significant inhibitory effect on postoperative aseptic inflammation.

(c) 2018 The Author(s)

Published by S. Karger AG, Basel

Xun Xu and Weilin Sang contributed equally to this work.

\section{Jinzhong $\mathrm{Ma}$}

Department of Orthopedics, Shanghai General Hospita

No. 650 Xinsongjiang Road

Shanghai, 201620 (China)

E-Mail majinzhong1963@sina.com 


\section{Introduction}

Osteoarthritis of the knee is a common disease that causes physical pain and dysfunction, thereby affecting human health and quality of life. Total knee arthroplasty (TKA) is an effective and widely used method for restoring joint function, reducing pain, correcting deformity, and improving the quality of life of patients. The criteria used to evaluate the effect of surgery focuses on 3 dimensions: inflammation, pain, and function, of which inflammation is the most important. However, aseptic inflammation often occurs at the surgical site postoperatively, leading to swelling and pain, thereby affecting patient recovery of joint function [1]. Holm et al. [2] found that after TKA, joint swelling caused by aseptic inflammation significantly impairs knee strength during early functional exercise, ultimately reducing the patients' $10-\mathrm{m}$ rapid walking ability by $57 \%$. However, despite such findings, previous clinical studies have not paid sufficient attention to postoperative assessment and to the treatment of aseptic inflammation. This oversight is mainly displayed in the lack of monitoring of aseptic inflammation and in the lack of assessment of the course and effects of anti-inflammatory treatment. Research shows that skin temperature, levels of C-reactive protein (CRP), erythrocyte sedimentation rate (ESR), and levels of interleukin-6 (IL-6) are effective indices for evaluating the degree of inflammation after surgery. Furthermore, local skin temperature and swelling degree typically reflect the extent of the local inflammatory reaction [3]. Cyclooxygenase-2 (COX-2) inhibitors inhibit prostaglandin production by inhibiting COX-2, and thus bring about a reliable anti-inflammatory and analgesic effect [4]. Therefore, this study compared an experimental group that used celecoxib and opioid analgesia with a control group that used only opioid analgesia within 6 weeks after TKA using skin temperature, CRP, ESR, and IL-6 levels as the primary means of monitoring inflammation to evaluate the anti-inflammatory effectiveness of celecoxib and its effects on the rehabilitation of joint function.

\section{Materials and Methods}

\section{Patients}

This work reports a prospective, randomized, controlled study; the study was approved by the ethics committee of Shanghai General Hospital and enrolled both male and female patients undergoing TKA during 2016-2017 in the Orthopedics Department of the hospital.
Patients who met all of the following standards were allowed to participate in this study: (1) a diagnosis in accordance with the diagnostic criteria of osteoarthritis; (2) the provision of a signed informed consent form, as well as the agreement and ability to comply with treatment plans, laboratory tests, and standard rehabilitation programs; (3) age <70 years; and (4) undergoing TKA completed by the same group of doctors and in accordance with the same standards. As part of this requirement, the prosthesis (bone cement prosthesis), surgical approach, tourniquet use, and placement of drainage tube and management remained the same. Patients were required to meet additional criteria, including the following: (1) a normal quadriceps muscle strength on the affected side; (2) no prior open surgery or fracture history on the affected side, with the current procedure being primarily for TKA; and (3) no patellar replacement performed during the surgery $[3,5-8]$.

The exclusion criteria were as follows: (1) the presence of infectious arthritis, diabetes, malignant tumors, autoimmune diseases or chronic inflammatory diseases; liver, kidney or blood system diseases; and an abnormal laboratory index (i.e., when the results of a liver function test was higher than twice the upper limit of normal, and the remaining indicators were not within the normal range); (2) severe osteoporosis; (3) severe bone deformity (the varus of the knee exceeds $30^{\circ}$ or the valgus of the knee exceeds $20^{\circ}$ ) or ligament insufficiency around the knee; (4) allergies to commonly used antibiotics; (5) high sensitivity to opioid drugs; (6) a history of ischemic heart disease or cerebrovascular disease (including coronary artery bypass surgery or stroke, cerebral ischemia, arrhythmia, heart failure, or myocardial infarction); (7) mental and nervous system diseases; (8) the presence of gastrointestinal perforation, ulcer, bleeding, or obstruction; (9) coagulation dysfunction and/or the long-term use of anticoagulant drugs; (10) allergies to celecoxib, sulpha drugs and/or aspirin; (11) use of hormone therapy; and (12) currently participating in other clinical trials or had participated in other clinical trials within the previous 3 months.

As part of the study protocol, it was decided that the participation of any subject would be terminated under any of the following circumstances: (1) the onset of severe perioperative complications, such as intra-articular infection, deep venous thrombosis of a lower limb, high fever of unknown cause or other complications during the rehabilitation process; (2) any adverse reactions to celecoxib during the observation period; (3) any clinical intervention affecting the outcome during the observation (e.g., the use of glucocorticoids); or (4) a request by the patient to withdraw from the research.

\section{Grouping}

We planned to include 60 patients at the start of the project. However, taking into account an estimated dropout rate of $20 \%$ during the follow-up period, we increased the sample size to 72 , with each group ultimately including 36 patients [9].

The selected patients were randomly divided into two groups as follows. (1) First, the subjects were successively numbered from 1 to 72 on the basis of the hospital admission time before the experiment. (2) Second, a random number was obtained by starting from an arbitrary number in a table of random numbers and taking a random number to correspond to each of the subjects along the same direction in the sequence. (3) Next, the random number was divided by the number of groups. If it was divisible, using the group number as a remainder. (4) Grouping was then completed according to the remainder value; that is, if the remainder was 1 , the pa- 
Fig. 1. Experimental design flow. Tramadol hydrochloride in both groups was given individually to ensure the patients can accept and complete a standardized rehabilitation exercise. The indices include white blood cell count, erythrocyte sedimentation rate, C-reactive protein, and interleukin-6 levels. PRN, as needed; ST, skin temperature; BID, twice daily; PO, oral; d, day; w, week.

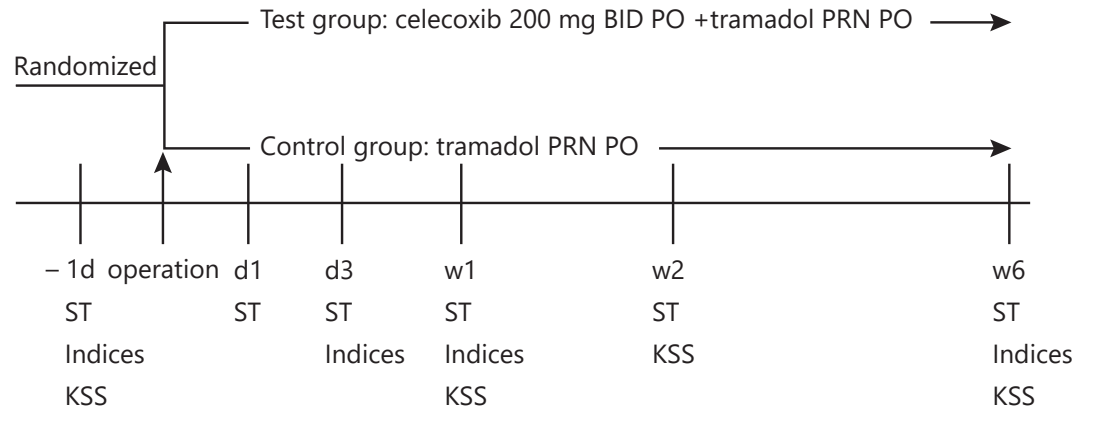

tient was assigned to the experimental group and if 2 , the patient was assigned to the control group. (5) If a group needed to be adjusted, 1 subject was needed to be extracted. Suppose the number of cases in the group was $N$, a random number was obtained and then divided by $N$. The subject, whose serial number was equal to the remainder (if it was divisible by $N$, then the remainder was $N$ ), was later moved to the other group until the 2 groups had the same number of cases $[6,7]$.

\section{Surgery}

The surgeries were all performed by the same group of doctors in accordance with the same standards. All patients were operated on under general anesthesia. First, a tourniquet was inflated to 240 $\mathrm{mm} \mathrm{Hg}$ before the surgery. Next, we made an anterior median incision and selected the medial patellar retinaculum approach. We cleaned the joint cavity after extensive exposure of the knee joint. With the osteotomy template, femoral condyle osteotomy was performed $6^{\circ}$ from the valgus and with an external rotation of approximately $3^{\circ}$. The tibial osteotomy was performed vertical to the tibial shaft, and hypokinesis was approximately $7^{\circ}$. During the surgery, patellar replacement was not performed, and the patella surface was formed. Next, an electric knife was used to cut the nerves around the patella. The bone cement prosthesis was installed after the irrigation gun was rinsed, and one drainage tube was placed, which was removed within $24 \mathrm{~h}$ after the surgery. Standardized nursing and rehabilitation training was given after the surgery, including the use of continuous passive motion (CPM), exercise guidance for the flexion and extension to improve function, and strength training guides for the quadriceps femoris.

\section{Drug Intervention Program}

Patients in the experimental group were treated with celecoxib $200 \mathrm{mg}$ twice daily per os and tramadol hydrochloride $50 \mathrm{mg}$ twice daily per os, while the control group was treated with tramadol hydrochloride $50 \mathrm{mg}$ twice daily per os. In both groups, the tramadol hydrochloride was given individually as required to ensure that the patients could accept and complete a standardized rehabilitation exercise. The dose started at $50 \mathrm{mg}$ but was allowed to reach $100 \mathrm{mg}$ as needed. The course of treatment was 6 weeks in total. Other medications remained the same in both groups; in other words, the following features remained the same - the anesthesia and related narcotic drug use, the local anesthesia (femoral nerve block), the preoperative use of $1.5 \mathrm{~g}$ cefuroxime to prevent infection with a continuance of routine use for 3 days after the sur- gery, and the postoperative use of rivaroxaban tablets $(10 \mathrm{mg}$, once daily per os) for 3 weeks.

\section{Experimental Design Flow}

We monitored changes in skin temperature around the knee and serum inflammatory indices as indicators of inflammation. Temperatures were measured using a portable digital thermometer with an accuracy of up to $0.2^{\circ} \mathrm{C}$. Temperatures were measured in the center of the patella and at 2, 4, 8, and 10 p.m., as well as the average. A similar measurement was also performed on the contralateral knee. When measuring the skin temperature, the measuring instrument was not placed directly in contact with the skin incision; instead, temperature measurements were recorded at a vertical distance of $1 \mathrm{~cm}$. There were no significant differences in the measurement time, room temperature or wound exposure time, and the patients were not trained or given ice compression for at least $1 \mathrm{~h}$ prior to measurement. Skin temperature was measured 1 day before surgery, on postoperative day 1 and day 3 , and at week 1 , week 2 , and week $6[3,5,6]$. We selected white blood cell (WBC) count, CRP and IL-6 levels, and ESR as blood monitoring indicators [10]. Inflammatory cytokines were measured preoperatively, on postoperative day 3 , and at week 1 and week 6 . At the same time, we scored the patients' knee using the original Knee Society Score (KSS). The KSS includes two components: a function score (KS-F) and a knee score, ranging from 0 to 100 points [11]. The knee score can be subdivided into two components, with 50 points allotted for one question that assesses both the frequency and severity of pain (KS-P) (50 points represents no pain) and the other 50 points for the clinical judgement (KS-C) of the ROM and stability of the joint. The function assessment rates the patient's walking distance and stair-climbing ability and makes deductions for the use of a walking aid. Research shows that among the $3 \mathrm{KS}$ subscales, $\mathrm{KS}-\mathrm{P}$ is the most responsive and $\mathrm{KS}-\mathrm{F}$ is the least responsive [12]. We chose the knee score, as the intensity of pain is proportional to the level of the score. KSS was rated preoperatively and at postoperative week 1 , week 2 , and week 6 (Fig. 1).

\section{Statistical Analyses}

All data are presented as means \pm standard error, and the graphical presentation was prepared using Graphpad Prism 5.0. Data. Student's $t$ test was used to analyze differences between the groups. $p<0.05$ was considered as indicating statistical significance. All statistical analyses were conducted using SPSS 20.0 statistical software (SPSS Inc., Chicago, IL, USA). 
Table 1. Skin temperature, WBC, CRP, ESR, and IL-6

\begin{tabular}{|c|c|c|c|c|c|c|c|}
\hline Indicator & Group & Preoperation & Day 1 & Day 3 & Day 7 & Week 2 & Week 6 \\
\hline \multirow[t]{2}{*}{ ST1 } & 1 & $34.2 \pm 0.19$ & $35 \pm 0.2$ & $34.2 \pm 0.2$ & $34 \pm 0.17$ & $33.4 \pm 0.21$ & $33.1 \pm 0.2$ \\
\hline & 0 & $34.1 \pm 0.17$ & $35.2 \pm 0.24$ & $34.5 \pm 0.22$ & $34.2 \pm 0.2$ & $33.8 \pm 0.17$ & $33.5 \pm 0.17$ \\
\hline \multirow[t]{2}{*}{ ST2 } & 1 & $34.6 \pm 0.19$ & $37.1 \pm 0.12^{*}$ & $37.3 \pm 0.16^{*}$ & $37 \pm 0.12^{*}$ & $36.2 \pm 0.16^{*}$ & $35.4 \pm 0.12 *$ \\
\hline & 0 & $34.7 \pm 0.18$ & $37.7 \pm 0.09$ & $37.7 \pm 0.12$ & $37.4 \pm 0.09$ & $36.6 \pm 0.12$ & $35.7 \pm 0.09$ \\
\hline \multirow[t]{2}{*}{ WBC } & 1 & $5.97 \pm 0.39$ & - & $8.39 \pm 0.54$ & $7.54 \pm 0.52$ & - & $6.25 \pm 0.21$ \\
\hline & 0 & $6.2 \pm 0.26$ & - & $7.81 \pm 0.49$ & $7.15 \pm 0.37$ & - & $6.33 \pm 0.19$ \\
\hline \multirow[t]{2}{*}{ CRP } & 1 & $3.2 \pm 1.23$ & - & $125.7 \pm 8.28$ & $26.4 \pm 2.12^{*}$ & - & $5 \pm 0.64^{*}$ \\
\hline & 0 & $3.7 \pm 1.8$ & - & $117.3 \pm 7.56$ & $45.7 \pm 4.13$ & - & $7.7 \pm 0.66$ \\
\hline \multirow[t]{2}{*}{ ESR } & 1 & $21 \pm 3.21$ & - & $74 \pm 4.2$ & $83 \pm 4.5$ & - & $43 \pm 3.06^{*}$ \\
\hline & 0 & $20 \pm 3.72$ & - & $68 \pm 5.56$ & $95 \pm 4.4$ & - & $60 \pm 3.42$ \\
\hline \multirow[t]{2}{*}{ IL-6 } & 1 & $4.56 \pm 0.6$ & - & $47.6 \pm 3.68$ & $15.78 \pm 1.13^{*}$ & - & $4.05 \pm 0.32$ \\
\hline & 0 & $8.81 \pm 6.1$ & - & $54.52 \pm 4.27$ & $22.39 \pm 2.08$ & - & $4 \pm 0.29$ \\
\hline
\end{tabular}

1, experimental group; 0, control group; ST1, skin temperature (in ${ }^{\circ} \mathrm{C}$ ) on the unaffected side; ST2, skin temperature of the affected side; WBC, white blood cells $\left(\times 10^{9} / \mathrm{L}\right)$; CRP, C-reactive protein $(\mathrm{mg} / \mathrm{L})$; ESR, erythrocyte sedimentation rate $(\mathrm{mm} / \mathrm{h})$; IL-6, interleukin-6 $(\mathrm{pg} / \mathrm{mL}){ }^{*} p<0.05$.

\section{Results}

Five patients in the experimental group and 3 in the control group were lost to follow-up because they could not be contacted. One patient in the experimental group stopped participation because he used proprietary Chinese medicine. Three in the control group were terminated because of vomiting (possibly a side effect of tramadol hydrochloride). A total of 60 cases were thus studied, with each group consisting of 30 cases. There were 13 males and 47 females. Of these 60 patients, 33 underwent right total knee arthroplasty and 27 underwent left total knee arthroplasty. The average age of the experimental group was $64.27 \pm 0.89$ years, and that of the control group was $64.47 \pm 0.68$ years. There was no significant difference between the groups ( $p=0.859$ ).

Patients were classified according to the X-ray Kellgren classification standard before the surgery. In the experimental group, 3 subjects were consistent with grade 2,19 with grade 3 , and 8 with grade 4 . In the control group, 4 subjects were consistent with grade 2, 16 with grade 3 , and 10 with grade 4 . There was no significant difference between the 2 groups $(p=0.838)$. All data are presented as means \pm standard error (Table 1 ).

Ipsilateral skin temperature, in both the control and experimental groups, reached a maximum on the 3rd day after TKA and began to decrease slowly thereafter; the temperature remained higher than the preoperative skin

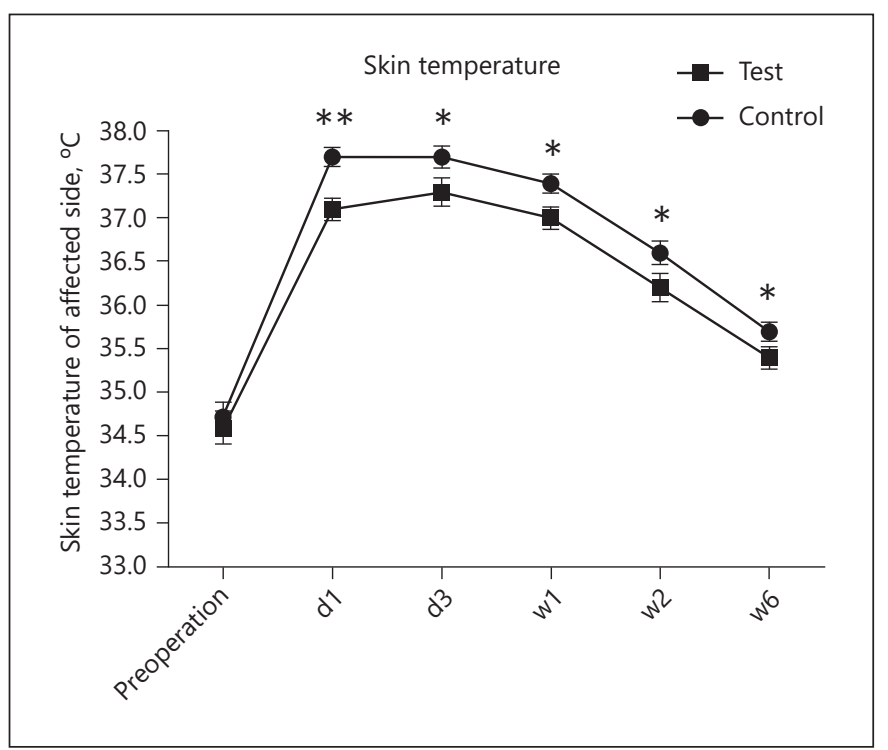

Fig. 2. Skin temperatures on the affected side. Except for the preoperative skin temperature, the recorded skin temperatures of the experimental group had statistically significant differences as compared with those of the control group $\left({ }^{* *} p<0.01\right.$, $\left.{ }^{*} p<0.05\right)$.

temperature until the 6th week (Fig. 2). The difference between preoperation and the 6 th week after operation in the experimental group was $0.8 \pm 0.2^{\circ} \mathrm{C}$, and that in the control group was $1.0 \pm 0.17^{\circ} \mathrm{C}(p<0.01$ for all $)$. The skin temperature in the ipsilateral side remained higher than 


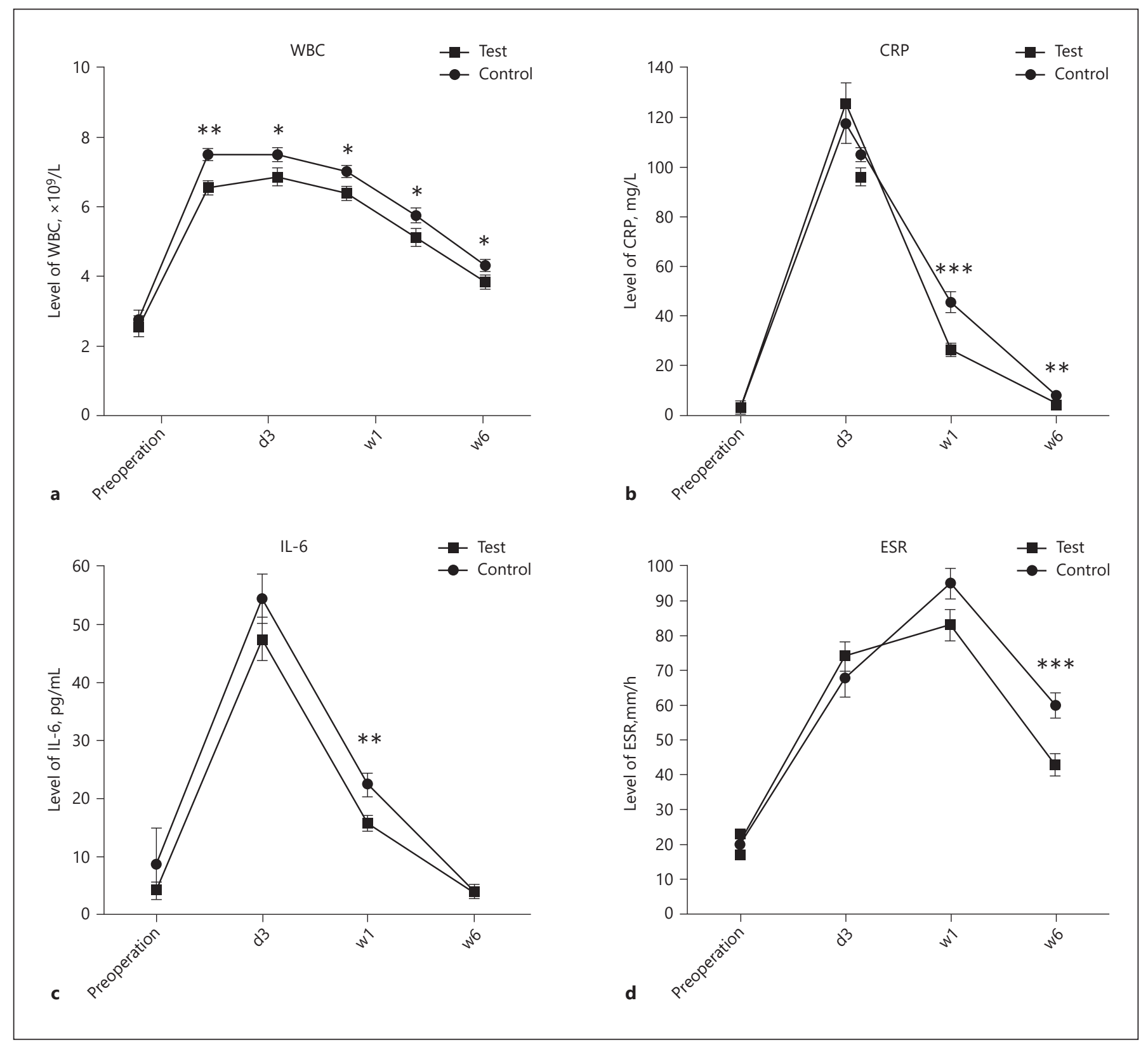

Fig. 3. Indices of inflammation. a WBC showed no significant differences at any point in the experiment. b Levels of CRP showed significant differences at the 1 st week and at the 6 th week after TKA $\left(* * p<0.01,{ }^{* * *} p<0.001\right)$. c Levels of IL- 6 were statistically different at the 1 st week after surgery $\left({ }^{* *} p<0.01\right)$. d ESR levels were different only after 6 th week after surgery $(* * * p<0.001)$.

that in the healthy side in the 6th week after surgery. The difference in the experimental group was $2.24 \pm 0.16^{\circ} \mathrm{C}$, and that in the control group was $2.15 \pm 0.14{ }^{\circ} \mathrm{C}(p<$ 0.001 ). Except for the preoperative skin temperature, the recorded skin temperatures in the experimental group were significantly different when compared with those in

Effect of Celecoxib on Surgical Site Inflammation the control group on the 1st and 3rd postoperative day, and at the 1st, 2nd, and 6th week after TKA ( $p=0.001$, $p=0.024, p=0.030, p=0.041$, and $p=0.047$, respectively).

WBC count, CRP, and IL-6 levels showed similar trends with skin temperature, which reached a maximum on the 3 rd postoperative day and decreased thereafter to 


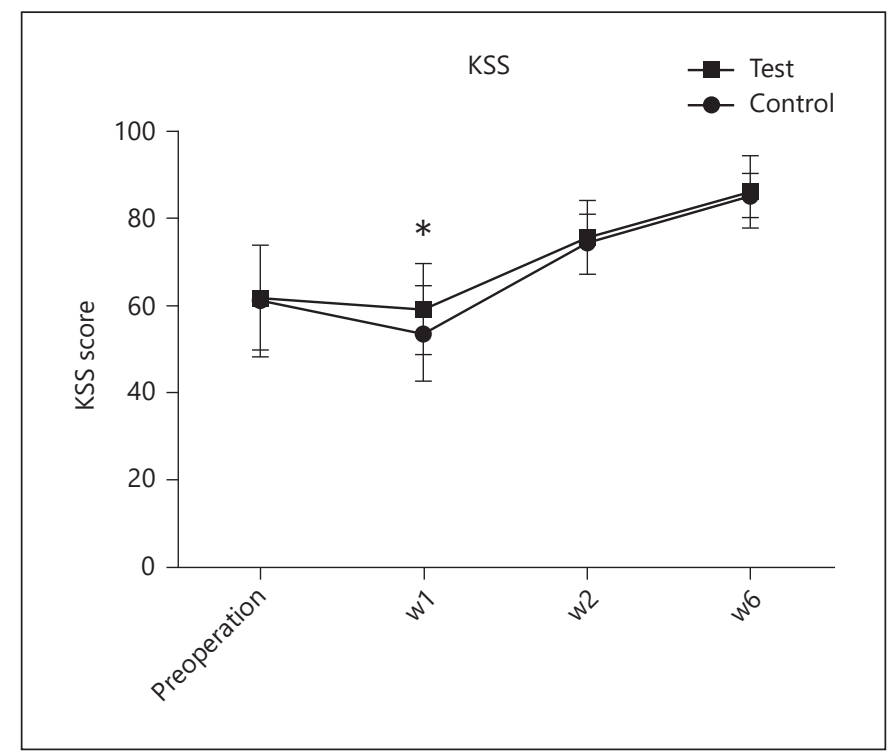

Fig. 4. The trend of the KSS. KSS were different at the 1st week after surgery $(* p<0.05)$.

the preoperative level at the 6th week after the surgery. WBC counts were not significantly different at any point during the experiment (Fig. 3a). Levels of CRP significantly differed at the 1 st week and at the 6th week after TKA ( $p=0.000$ and $p=0.006$, respectively) with values of $19.3 \pm 4.64 \mathrm{mg} / \mathrm{L}$ and $2.6 \pm 0.92 \mathrm{mg} / \mathrm{L}$, respectively (Fig. 3b). Levels of IL-6 significantly differed at the 1st week after surgery $(6.61 \pm 2.36 \mathrm{pg} / \mathrm{mL}, p=0.007)$ (Fig. $3 \mathrm{c}$ ). ESR demonstrated a different trend than the above indices; ESR increased to its maximum at the 1st week postoperatively and remained higher than its preoperative level at the 6th week, at which time it was $22 \pm 3.58 \mathrm{~mm} / \mathrm{h}$ higher in the experimental group and $39 \pm 4.0 \mathrm{~mm} / \mathrm{h}$ higher in the control group $(p<0.01)$. Not until after the 6th postoperative week did the ESR show a difference (17 $\pm 4.6 \mathrm{~mm} / \mathrm{h}$ between the two groups, $p=0.001$ ) (Fig. $3 \mathrm{~d}$ ).

KSS scores differed at the 1 st week after surgery $(p=$ 0.049) (Fig. 4).

\section{Discussion}

Inflammatory markers (WBC count, ESR, and levels of CRP and IL-6) are important indicators of inflammation that can effectively be used to evaluate infection. IL-6 is an especially sensitive indicator of inflammation $[10,13]$. In this study, the levels of WBC, CRP, and IL- 6 in the two groups were highest on the 3rd day after surgery and re- turned to their preoperative levels by the 6th week after surgery. However, ESR exhibited an increasing trend on the $3 \mathrm{rd}$ day, reached a maximum during the 1 st week, and declining slowly thereafter; however, levels remained higher at the 6th week than preoperative levels. WBC count, levels of CRP, and IL- 6 seem to be more sensitive to inflammation than ESR. This study does not provide information on the time point at which ESR decreases to preoperative levels. It has been reported previously that IL-6 levels reach a maximum of $140 \mathrm{pg} / \mathrm{mL} 6 \mathrm{~h}$ after surgery and are reduced to less than $50 \mathrm{pg} / \mathrm{mL} 48-72 \mathrm{~h}$ after surgery [14]. We measured IL-6 at longer intervals, and the "maximal" levels detected by us may not be the actual maximum. However, its level was identical to that observed in previous studies. IL-6 may have a higher sensitivity to the inflammatory response, but its level remained higher than its preoperative level at the 1st week after surgery. Unfortunately, due to the lack of earlier data, we did not observe the true maximum value of IL-6. None of the patients in this study had infections; therefore, the changes in inflammatory factors indicated that the increase in levels of inflammatory factors after surgery was a normal reaction, representing the necessary process of self-protection and restoration after the body experiences trauma. However, when the inflammatory factors show a long-term increase, it is important to be alert for postoperative infection.

There were no significant differences between the inflammatory factors on the 3rd day after surgery. The inflammatory reaction reached a maximum on the 3rd day, and the intensity of the inflammatory response was far greater than that of the anti-inflammatory response, which resulted in a lack of significant differences. Levels of CRP and IL-6 were significantly lower in the experimental group than in the control group at the 1st week. At the 6th week after surgery, levels of CRP and ESR in the experimental group were lower than those in the control group. At the same time, IL-6 was reduced to its preoperative level and did not significantly differ between the 2 groups. However, the observed decrease to preoperative levels does not necessarily mean that no aseptic inflammatory reaction occurred. ESR remained higher than the preoperative level. Furthermore, many researchers have reported levels of CRP, ESR, and IL-6 and found that the levels of these inflammatory factors in patients with osteoarthritis were higher than those normally present in the blood $[15,16]$. Thus, although the aseptic inflammatory reaction was reduced significantly, it was still present.

This study shows that celecoxib exerts a definite antiinflammatory effect by significantly accelerating a de- 
crease in the levels of inflammatory factors, especially during the early postoperative period.

The increase in skin temperature at inflammatory sites is well known; experienced surgeons touch the skin to determine the onset of infection. However, there remains no objective and effective way to monitor postoperative skin temperatures at the surgical site. In our experiment, the skin temperature reached a maximum on the 3rd day and decreased thereafter, showing the same trends with time as WBC count, and levels of CRP and IL-6. Skin temperature might indicate the strength of the inflammatory reaction. Six weeks after the surgery, the skin temperature on the affected side remained higher than that measured before the surgery $(p<0.001)$. With the exception of ESR, the studied inflammatory factors had decreased to preoperative levels by the end of the measurement period. In conclusion, skin temperature appeared to be more sensitive in reflecting local inflammatory status than the levels of inflammatory factors in the peripheral blood. In addition, there were significant differences in skin temperature between the experimental and control groups from the 1st postoperative day to the 6 th week after surgery, indicating that skin temperature is more sensitive than levels of inflammatory factors in the peripheral blood for measuring local inflammatory reactions. The skin temperature of the affected knee remained higher than that on the contralateral side at the 6 th week. Further research is needed to determine the time point after surgery when the skin temperatures on the healthy and affected side are no longer different from each other. However, for clinicians it is clear that surgical sites typically continue to experience inflammation 6 weeks after surgery; at this time, the skin temperature on the affected side has not decreased to the preoperative level and remains approximately $2{ }^{\circ} \mathrm{C}$ higher than that on the contralateral side; thus, when the skin temperature is within this range perhaps we need not be concerned about postoperative infection. However, for skin temperature to replace invasive blood tests as a reliable and noninvasive indicator of inflammation and infection would require further multicenter studies on large sample sizes to clarify its correlation with inflammatory factors.

The KSS significantly differed at the 1st week after surgery. There were no significant differences between the experimental and control groups at the $2 \mathrm{nd}$ and 6 th week after surgery, although the score in the experimental group was higher than that in the control group. The difference was mainly caused by resting pain and joint activity in the 1st week. Resting pain was lower, and fewer pa- tients complained of resting pain in the experimental group than in the control group. Joint activity, especially the flexion angle, was larger in the experimental group. The use of a selective COX-2 inhibitor benefited early functional recovery by inhibiting the inflammatory response. At the 2nd and 6th week after surgery, there were no significant differences between the 2 groups; we suggest that this was because the maximum inflammatory response had ended, the pain of the patients was reduced, and the patients were better able to begin active functional exercise.

This study has certain deficiencies. This was a singlecenter study, and the sample size is not large. It is difficult to control variables in clinical research. It is particularly different to control the variable of temperature measurement. Larger sample sizes are needed to reduce errors and enhance the credibility of the results. Also, more long-term monitoring is needed to ascertain the time points when ESR and skin temperature drop to preoperative levels.

\section{Conclusions}

The results of this study indicate that the use of a selective COX-2 inhibitor has a significant inhibitory effect on the postoperative aseptic inflammatory reaction, especially during the early period, and that the decrease in inflammatory response is beneficial to the prognosis of patients. Specifically, the use of COX-2 inhibitor can promote the early rehabilitation of knee joint function during the early stage. Additionally, the exorbitant postoperative inflammatory response can lead to immune suppression [17]. Studies have also shown that a close relationship exists between the aseptic loosening of a prosthesis and inflammatory factors after TKA [18]. Therefore, whether intended for patients with early postoperative rehabilitation or for a long-term curative effect, postoperative anti-inflammatory treatments are conducive not only to analgesia but also to obtaining a longterm stable knee joint. In addition, the false-negative rate of white blood cells is high, and this indicator should be combined with IL-6, CRP and other inflammatory indices. Understanding changes in the postoperative inflammatory reaction will be of great help to clinicians for evaluating the status of patients. In conclusion, clinicians should pay attention not only to postoperative infection but also to the monitoring and evaluation of the postoperative aseptic inflammatory response, and active measures should be taken. 


\section{Acknowledgment}

This study is sponsored by Pfizer. This study is supported by Shanghai General Hospital (NO.0703N16016) and Shanghai Municipal Health and Family Planning Commission (No. 20164Y0270).

\section{References}

1 Feng Y, Ju H, Yang B, An H: Effects of a selective cyclooxygenase- 2 inhibitor on postoperative inflammatory reaction and pain after total knee replacement. J Pain 2008;9:44-52.

2 Holm B, Kristensen MT, Bencke J, Bencke J, Husted H, Kehlet H, et al: Loss of knee-extension strength is related to knee swelling after total knee arthroplasty. Arch Phys Med Rehabil 2010;91:1770-1776.

3 Honsawek S, Deepaisarnsakul B, Tanavalee A, Sakdinakiattikoon M, Ngarmukos S, Preativatanyoum K, et al: Relationship of serum IL-6, C-reactive protein, erythrocyte sedimentation rate, and knee skin temperature after total knee arthroplasty: a prospective study. Int Orthop 2011;35:31-35.

4 Cashman JN: The mechanisms of action of NSAIDs in analgesia. Drugs 1996;9:13-23.

5 Mingjiang M, Shake Y, Xindie Z, Rongxin H: Value of knee skin temperature measured by infrared thermography and soluble intercellular adhesion molecule- 1 in the diagnosis of peri-prosthetic knee infection in Chinese individuals following total knee arthroplasty. Chin Med J (Engl) 2014;127:3105-3109.

6 Windisch C, Brodt S, Roehner E, Matziolis G: Regional differences in temperature course after knee arthroplasty. Knee Surg Sports Traumatol Arthrosc 2016;24:2686-2691.

\section{Disclosure Statement}

The authors declare no conflicts of interest.
7 Schroer WC, Diesfeld PJ, LeMarr AR, Reedy ME: Benefits of prolonged postoperative cyclooxygenase-2 inhibitor administration on total knee arthroplasty recovery: a doubleblind, placebo-controlled study. J Arthroplasty $2011 ; 26: 2-7$.

8 lvarez-Soria MA, Largo R, Santillana J, Sanchez-Pernaute O, Calvo E, Hernandez M, et al: Long term NSAID treatment inhibits COX-2 synthesis in the knee synovial membrane of patients with osteoarthritis: differential proinflammatory cytokine profile between celecoxib and aceclofenac. Ann Rheum Dis 2006;65:998-1005.

9 Gupta KK, Attri JP, Singh A, Kaur G: Basic concepts for sample size calculation: critical step for any clinical trials! Saudi J Anaesth 2016;10:328-331.

10 Drago L, Vassena C, Dozio E, Corsi MM, De Vecchi E, Mattina R, Romanò C: Procalcitonin, C-reactive protein, interleukin-6, and soluble intercellular adhesion molecule- 1 as markers of postoperative orthopaedic joint prosthesis infections. Int J Immunopathol Pharmacol 2011;24:433-440.

11 Insall JN, Dorr LD, Scott RD, et al: Rationale of the Knee Society clinical rating system. Clin Orthop Relat Res 1989;248:13-14.

12 Ko Y, Lo N-N, Yeo S-J, Yang K-Y, Yeo W, Chong $\mathrm{H}-\mathrm{C}$, et al: Comparison of the responsiveness of the SF-36, the Oxford Knee Score, and the Knee Society Clinical Rating System in patients undergoing total knee replacement. Qual Life Res 2013;22:2455-2459.
13 Lisowska B, Mamlijski W, Maidyk P, Zabek J, Baranowska E: The role of cytokines in inflammatory response after total knee arthroplasty in patients with rheumatoid arthritis. Rheumatol Int 2008;28:667-671.

14 Wirtz DC, Heller KD, Miltner O, Zilkens $\mathrm{K}-\mathrm{W}$, Wolff JM: Interleukin-6: a potential inflammatory marker after total joint replacement. Int Orthop 2000;24:194-196.

15 Berenbaum F, Eymard F, Houard X: Osteoarthritis, inflammation and obesity. Curr Opin Rheumatol 2013;25:114-118.

16 Sanchez-Ramirez DC, van der Leeden M, van der Esch M, Gerritsen M, Roorda LD, Verschueren S, et al: Association of serum C-reactive protein and erythrocyte sedimentation rate with muscle strength in patients with knee osteoarthritis. Rheumatology 2013;52: 727-732.

17 Menger MD, Vollmar B: Surgical trauma: hyperinflammation versus immunosuppression? Langenbecks Arch Surg 2004;389:475484.

18 Dasa V, Kramer JM, Gaffen SL, Kirkwood KL, Mihalko WM: Is monocyte chemotactic protein 1 elevated in aseptic loosening of TKA? Clin Orthop Relat Res 2012;470:1879-1884. 